\title{
Mechanisms of action of adjuvants
}

\author{
Sunita Awate ${ }^{1,2}$, Lorne A. Babiuk ${ }^{3}$ and George Mutwiri ${ }^{1,2 *}$ \\ 1 Vaccine and Infectious Disease Organization-International Vaccine Centre, School of Public Health, University of Saskatchewan, Saskatoon, SK, Canada \\ ${ }^{2}$ Vaccinology and Immunotherapeutics program, School of Public Health, University of Saskatchewan, Saskatoon, SK, Canada \\ ${ }^{3}$ University of Alberta, Edmonton, AB, Canada
}

\section{Edited by:}

Volker Gerdts, Vaccine and Infectious Disease Organization-International

Vaccine Centre, Canada

Reviewed by:

Sylvie Fournel, Strasbourg University, France

Paola Massari, Boston University, USA

*Correspondence:

George Mutwiri, Vaccine and Infectious Disease

Organization-International Vaccine Centre, School of Public Health,

University of Saskatchewan, 120

Veterinary Road, Saskatoon, SK S7N

5E3, Canada.

e-mail: george.mutwiri@usask.ca
Adjuvants are used in many vaccines, but their mechanisms of action are not fully understood. Studies from the past decade on adjuvant mechanisms are slowly revealing the secrets of adjuvant activity. In this review, we have summarized the recent progress in our understanding of the mechanisms of action of adjuvants. Adjuvants may act by a combination of various mechanisms including formation of depot, induction of cytokines and chemokines, recruitment of immune cells, enhancement of antigen uptake and presentation, and promoting antigen transport to draining lymph nodes. It appears that adjuvants activate innate immune responses to create a local immuno-competent environment at the injection site. Depending on the type of innate responses activated, adjuvants can alter the quality and quantity of adaptive immune responses. Understanding the mechanisms of action of adjuvants will provide critical information on how innate immunity influences the development of adaptive immunity, help in rational design of vaccines against various diseases, and can inform on adjuvant safety.

Keywords: adjuvants, mechanisms, innate immunity, cell recruitment and activation, inflammasomes, antigen presentation, dendritic cells

\section{INTRODUCTION}

The goal of vaccination is induction of protective immunity and in some vaccines this can be enhanced by addition of adjuvants. Adjuvants (Latin word adjuvare, meaning "to help or aid") were first described by Ramon as "substances used in combination with a specific antigen that produced a more robust immune response than the antigen alone" (Ramon, 1924). Many diverse classes of compounds have been assessed as adjuvants including mineral salts, microbials products, emulsions, saponins, cytokines, polymers, microparticles, and liposomes (Guy, 2007). Based on their proposed mechanisms of action, vaccine adjuvants have been broadly divided into delivery systems and immunostimulatory adjuvants (Singh and O'Hagan, 2003). In general, delivery systems were previously thought to act by providing a depot while immuno-stimulatory adjuvants activate cells of the innate immune system (Pashine et al., 2005). However, this classification is no longer appropriate since now there is evidence that some delivery systems can activate innate immunity.

Surprisingly, despite the wide use of vaccine adjuvants in billions of doses of human and animal vaccines, the mechanisms of action by which they potentiate immune responses are not well characterized. This is well captured in a famous quote by Janeway (1989) who observed that adjuvants are "the immunologists' dirty little secret." However, recent advances in immunobiological research have revealed several mechanisms by which adjuvants act. Available evidence suggests that adjuvants employ one or more of the following mechanisms to elicit immune responses: (1) sustained release of antigen at the site of injection (depot effect), (2) up-regulation of cytokines and chemokines, (3) cellular recruitment at the site of injection, (4) increase antigen uptake and presentation to antigen presenting cells (APC), (5) activation and maturation of APC [increased major histocompatibility complex (MHC) class II and co-stimulatory molecules expression] and migration to the draining lymph nodes, and (6) activation of inflammasomes (Figure 1) (Cox and Coulter, 1997; Hoebe et al., 2004; Fraser et al., 2007). In this review, we will address the proposed mechanisms of action of vaccine adjuvants with specific emphasis on licensed adjuvants (Table $\mathbf{1}$ ).

\section{FORMATION OF DEPOT AT THE SITE OF INJECTION}

The formation of a depot at the injection site is perhaps the oldest and most widely recognized mechanism of action of adjuvants. Antigen trapping and slow release at the site of injection ensures constant stimulation of the immune system for production of high antibody titers (Siskind and Benacerraf, 1969). Until recently, depot effect was considered a classic mechanism of action of many adjuvants. Glenny et al. (1926) were the first to propose the importance of depot formation in the adjuvant activity of alum. Antigen was detected for 2-3 weeks in alumina gel-induced granulomas (Osebold, 1982). Antigens are simply adsorbed onto the alum but the binding is proposed to be due to strong electrostatic interaction between antigen and alum (Burrell et al., 2000), which enhanced antigen uptake and presentation by APCs (Mannhalter et al., 1985). Various other adjuvants such as water-in-oil emulsions [Complete Freunds Adjuvant (CFA)] and biodegradable micro-and nano-particles were shown to act by depot effect to generate prolonged and sustained high antibody titers (Herbert, 1968; Kreuter, 1988). AS04, an adjuvant combination consisting of monophosphoryl lipid A (MPL) and alum was shown to induce optimal immune responses only when co-localized with antigen (Didierlaurent et al., 2009). The presence of alum in AS04 is important in stabilizing the MPL and antigen within the 


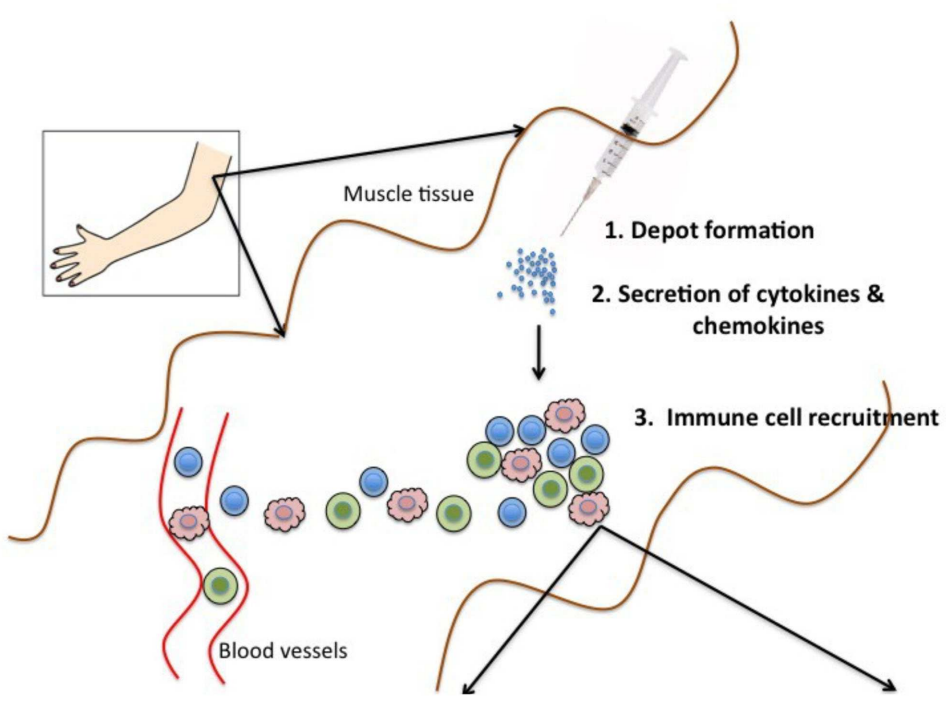

5. Maturation and activation of immune cells (co-stimulatory molecule expression)
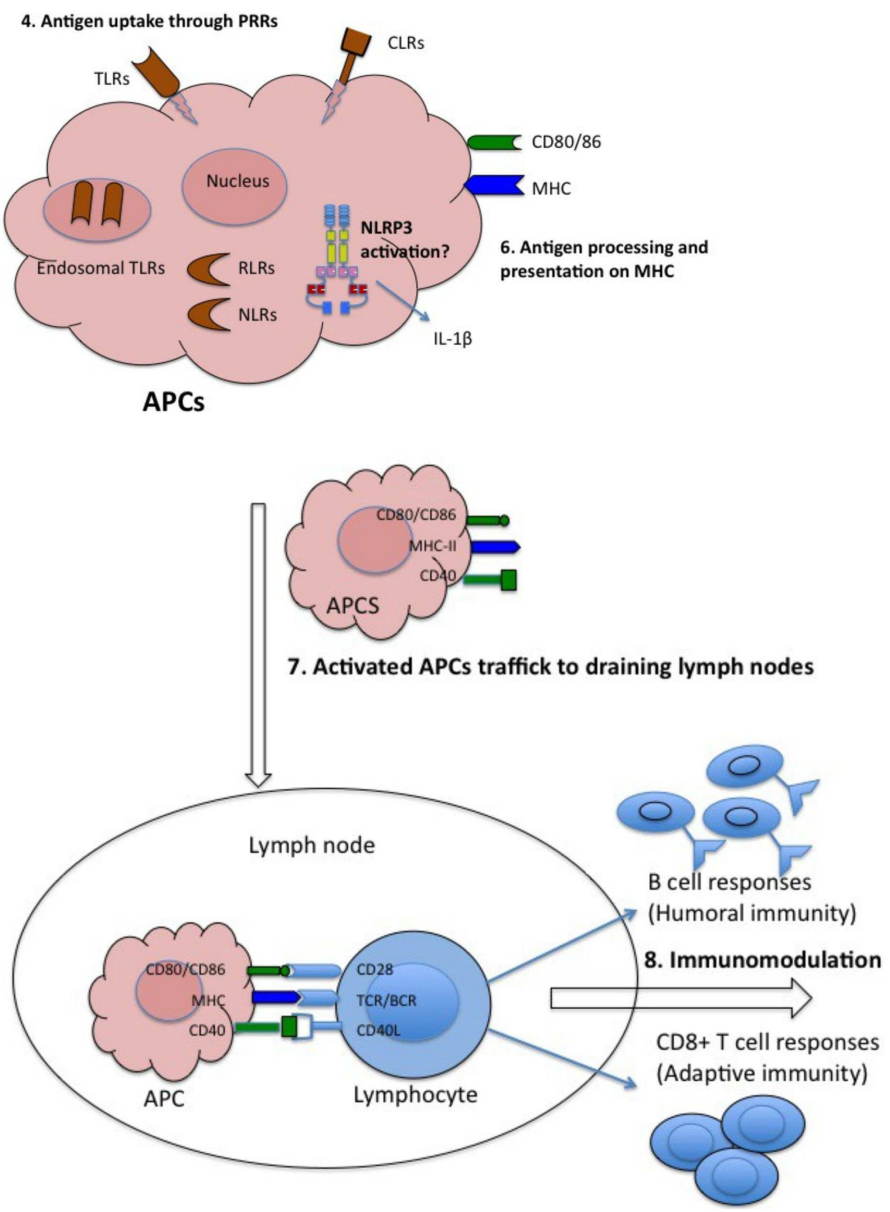

FIGURE 1 | Proposed mechanisms of action of adjuvants. (1) Some adjuvants presumably form a depot at the site of injection, which is associated with slow release of antigen. (2) Other adjuvants are associated with transient secretion of cytokines and chemokines. (3) Secreted cytokines and chemokines are involved in recruitment of various immune cells to the injection site. These recruited cells secrete cytokines and chemokines, in turn attract other immune cells. All these events lead to formation of a local immuno-competent environment at the injection site. (4) The recruited APCs express various PRRs both on the surface (TLRs, CLRs) and intracellularly (NLRs and RLRs), which are recognized and/or are activated by the adjuvants. (5) This leads to maturation and activation of recruited APCs. Mature APCs up-regulate the expression of $\mathrm{MHC}$ and co-stimulatory molecules. (6) They are also characterized by increased capacity for antigen processing and presentation. (7) Mature APCs then migrate to the draining lymph nodes to interact with antigen-specific B or T cell to (8) activate potent antibody secreting $\mathrm{B}$ cells and/or effector $\mathrm{CD}^{+} \mathrm{T}$ cell responses. 
Table 1 | Mechanisms of action of adjuvants licensed for human use.

\begin{tabular}{lllll}
\hline $\begin{array}{l}\text { Adjuvants } \\
\text { Plum }\end{array}$ & $\begin{array}{l}\text { Immune response } \\
\text { action }\end{array}$ & Licensed vaccines & Reference \\
\hline No depot effect & $\uparrow$ Ab responses & $\begin{array}{l}\text { Many human vaccines (e.g., DTap, } \\
\text { Hib, Hepatitis A, Hepatitis B) }\end{array}$ & $\begin{array}{l}\text { Gavin et al. (2006), Franchi and } \\
\text { Nüez (2008), Kool et al. (2008a), } \\
\end{array}$ \\
& & & McKee et al. (2009), Hutchison \\
& & & et al. (2012)
\end{tabular}

Independent of TLR signaling $\uparrow$ Local cytokines and chemokines $\uparrow$ Cell recruitment (eosinophils, monocytes, macrophages) $\uparrow$ Ag presentation

MF59 No depot effect

NLRP3 independent but ASC-dependent Independent of TLR signaling but MyD88-dependent for Ab responses $\uparrow$ Local cytokines and chemokines $\uparrow$ Cell recruitment (neutrophils, macrophages, and monocytes) $\uparrow$ Ag uptake Activate muscle cells $\uparrow$ Ag-loaded neutrophils and monocytes in dLNs

AS04 MPL signals through TLR4 to activate APCs

$\uparrow$ Local cytokines and chemokines $\uparrow$ Cell recruitment (DCs and monocytes) $\uparrow$ Ag-loaded DCs and monocytes in dLNs

Spatio-temporal co-localization with $\mathrm{Ag}$ Transient $\uparrow$ cytokines locally and in $\mathrm{dLNs}$

$\uparrow$ Cell recruitment (granulocytes and monocytes)

$\uparrow$ Ag-loaded monocytes in dLNs

Bind APCs and induce

$\uparrow \mathrm{CTL}$ responses

$\uparrow$ Th2 responses

Poor Th1 responses

Balanced Th1 and Th2 responses

$\uparrow \mathrm{Ab}$ responses

$\uparrow A b$ responses

$\uparrow$ Immune memory

receptor-mediated endocytosis

Escape endosomal degradation

Ag presentation via MHC class II and

$\mathrm{MHC}$ class I to CD4+ T cells and

$\mathrm{CD} 8+\mathrm{T}$ cells respectively

Immunopotentiator
Licensed for influenza vaccine (Fluad $\left.^{\circledR}\right)$, H5N1 pre-pandemic vaccine (Aflunov ${ }^{\circledR}$ ), H1N1 pandemic vaccines (Focetria ${ }^{\circledR}$ and Celtura ${ }^{\circledR}$ )
Dupuis et al. (1999), Mosca et al. (2008), Calabro et al. (2011), Ellebedy et al. (2011)
Licensed for human papilloma virus (HPV) (Cervarix ${ }^{\mathrm{TM}}$ ), hepatitis $\mathrm{B}$ virus (Fendrix ${ }^{\circledR}$ )
Licensed for pandemic flu vaccine Morel et al. (2011) (Pandemrix ${ }^{\circledR}$ )
Licensed for Inflexal ${ }^{\circledR} \mathrm{V}$ and Invivac ${ }^{\circledR}$ influenza vaccine and hepatitis $A$ vaccines (Epaxal ${ }^{\circledR}$ )
Glück et al. (1992), Bungener et al. (2002a, b), Khoshnejad et al. (2007)

$A b$, antibody; Ag, antigen; CTL, cytotoxic T lymphocytes; dLNs, draining lymph nodes. 
vaccine, along with providing a depot effect (Didierlaurent et al., 2009). The cationic adjuvant formulation (CAF) 01, a combination of dimethyldioctadeclammonium/trehalose-6,6-dibehenate (DDA/TDB), which is currently in phase I clinical trial, is also thought to induce long lasting depot effect (Henriksen-Lacey et al., 2010).

There is no definitive evidence that depot effect significantly contributes to adjuvant activity (Marrack et al., 2009). In various studies, it has been shown that surgical removal of the antigenalum depot 14 days after immunization had no effect on the immune responses (Schijns, 2000). Apparently, the adsorption of antigen to alum was not required for alum adjuvant activity (Iyer et al., 2003; De Gregorio et al., 2008). It was recently reported that removal of the injection site $2 \mathrm{~h}$ after antigen and alum administration had no effect on humoral or cell-mediated immunity (Hutchison et al., 2012). Similarly, MF59 was rapidly cleared and did not form a depot at the injection site (Ott et al., 1995). MF59 was distributed and cleared independent of antigen with a half-life of $42 \mathrm{~h}$ in the muscle tissue (Dupuis et al., 1999). Likewise, ISCOMs tend to be rapidly transported to the draining lymph nodes after administration (Morein and Bengtsson, 1999). Together, these studies clearly indicate that depot effect is not required for adjuvant activity of alum, and possibly MF59 or ISCOMs.

\section{UP-REGULATION OF CYTOKINES AND CHEMOKINES LEADING TO CELLULAR RECRUITMENT AT THE INJECTION SITE}

Recent studies on the mechanisms of adjuvants have focused on recruitment of innate immune cells at the site of injection. Particulate adjuvants have been shown to create a local pro-inflammatory environment to recruit immune cells (Goto and Akama, 1982). Using genome wide microarray analysis, Mosca et al. (2008) demonstrated that a cluster of genes encoding cytokines, chemokines, innate immune receptors, interferoninduced genes, and gene encoding adhesion molecules defined as "adjuvant core response genes" were commonly modulated by alum, MF59, and CpG-ODN at the site of injection. Compared with alum and CpG-ODN (TLR9 agonist), MF59 was a strong modulator of adjuvant core response genes. Chemokines, which play a critical role in tissue specific migration of immune cells, were shown to be up-regulated by adjuvants at the injection site. MF59 significantly up-regulated the expression of CCR2, a receptor for CCL2, which is involved in monocyte infiltration. This was in agreement with previous in vitro results showing that MF59 induced release of chemo-attractants like CCL2, CCL3, CCL3 and CXCL8 (Seubert et al., 2008). Further, studies in CCR2-deficient mice showed that MF59-induced mononuclear cell recruitment is CCR2 dependent (Dupuis et al., 2001). Another oil-in-water emulsion AS03 co-localizes with antigen to trigger colony-stimulating factor 3 (CSF3) and IL-6, and leukocyterecruiting chemokines CCL2, CCL3, and CCL5 at the site of injection (Morel et al., 2011). Similar cytokine and chemokine mRNA expression profiles were up-regulated in the draining lymph nodes (Morel et al., 2011). Likewise, alum-induced infiltration of immune cells was accompanied by production of chemoattractants like CCL2, the neutrophil chemotaxin KC (CXCL1), and eosinophil chemotaxin eotaxin (CCL11) in the peritoneal cavity of mice (Kool et al., 2008b). Similarly, a novel adjuvant, poly[di(sodiumcarboxylatoethylphenoxy)phosphazene] (PCEP), induced stronger expression of adjuvant core response genes compared to CpG at the site of injection. Locally, PCEP triggered production of pro-inflammatory cytokines and chemokines including CCL2 (Awate et al., 2012).

Alum promotes Th2-type immune responses and differentiation of B cells resulting in robust antibody production (Grun and Maurer, 1989). However, the role of Th2 cytokines in the adjuvant activity of alum is not clearly defined. In vitro studies indicate that alum-induced activation of macrophages and up-regulation of co-stimulatory molecules did not depend on IL-4 (Rimaniol et al., 2004). However, in in vivo studies, alum-induced priming of B cells through IL- 4 producing $\mathrm{Gr}^{+}{ }^{+}$cells in mouse spleen, which is required for proliferation of antigen-specific B cells and for optimal antibody production (Jordan et al., 2004). IL-4 producing $\mathrm{Gr}^{+}$cells were mainly eosinophils, which appeared within $24 \mathrm{~h}$ and induced expansion of B cells and enhanced IgM production (Wang and Weller, 2008). Further, studies with eosinophildeficient mice showed that the priming of B cells was abolished after alum injection confirming the central role of eosinophils in alum-induced Th2-type immune responses (Jordan et al., 2004; Wang and Weller, 2008). In addition, a study by Serre et al. (2008) revealed that the Th2-type immune responses generated by alum may signal through IL-25/IL-17RB and/or IL-6 pathways.

Alum has been shown to activate the complement cascade and recruit cells from blood to create an inflammatory environment at the site of injection (Ramanathan et al., 1979; Goto et al., 1997). Similar to alum, MF59 has been shown to recruit CD11b ${ }^{+}$ blood mononuclear cells in the mouse muscle (Mosca et al., 2008). Intra-peritoneal injection of alum-induced rapid cell recruitment of inflammatory $\mathrm{Ly}_{6} \mathrm{C}^{+} \mathrm{CD} 1 \mathrm{bb}^{+}$monocytes. The inflammatory monocytes take up antigen, differentiate into $\mathrm{CD}_{11 \mathrm{c}^{+}} \mathrm{MHC}$ class $\mathrm{II}^{+}$DCs in a myeloid differentiation primary response gene 88 (MyD88)-dependent manner and migrate to draining lymph nodes, where they induced proliferation of antigen-specific T cells (Kool et al., 2008a). In similar studies by McKee et al. (2009), aluminduced rapid recruitment of various polymorphonuclear (PMN) cells including eosinophils, monocytes, neutrophils, DCs, natural killer (NK), and NKT cells at the site of vaccination. Interestingly, in cell depletion studies in mice, alum-mediated humoral and cellular responses were independent of mast cells, macrophages, and of eosinophils (McKee et al., 2009).

MF59-mediated immune cell recruitment to the injection site has been studied in detail (Calabro et al., 2011). MF59 induced recruitment of neutrophils, monocytes, eosinophils, macrophages followed by DCs after i.m. injection in mice. The recruited cells especially neutrophils, monocytes, and B cells take up both antigen and adjuvant and traffick to draining lymph nodes. Neutrophils are the first cells to be recruited at the site of adjuvant injection and also one of the highest in numbers. However, depletion of neutrophils had no impact on the antigen-specific immune responses induced by MF59 (Calabro et al., 2011). Similar to MF59, administration of AS03 led to enhanced recruitment of neutrophils, eosinophils, and monocytes at the site of injection, which take up antigen and traffick to draining lymph nodes (Morel et al., 2011). 
At the injection site, neutrophils attract other immune cells by producing increased amounts of chemokines and transport antigen to the draining lymph nodes (Calabro et al., 2011; Morel et al., 2011). However, the role of neutrophils in adjuvant activity is not completely clear.

ASO4 induces transient local NFאB activity and cytokine production (Didierlaurent et al., 2009). The TLR4 agonist MPL, one of the components of AS04, stimulated increased numbers of DCs and monocytes in the draining lymph nodes. Likewise $\mathrm{CpG}$, a TLR9 agonists, signals through activation of MyD88, IRAK, and TRAF-6, leading to recruitment of transcriptional factors, which in turn up-regulates the pro-inflammatory genes and protein expression (IL-1, IL-6, IL-12, IL-18, and TNF- $\alpha$ ) within $3 \mathrm{~h}$ of injection (Klinman et al., 1996; Klaschik et al., 2009). Genes up-regulated by CpG included cytokines, cell signaling, cell movement, and DNA damage response genes (Klaschik et al., 2010). One of the roles of cationic liposomes is to recruit immune cells and increase antigen presentation. Intra-peritoneal injection of cationic liposome (DDA/MPL) increases influx of neutrophils, monocytes, macrophages, and activated NK cells in the peritoneal cavity (Korsholm et al., 2010). Another cationic liposome CAF01 induced recruitment of monocytes to the site of injection and increased trafficking of liposomes to the draining lymph nodes (Henriksen-Lacey et al., 2010).

Therefore, adjuvants induce recruitment of various immune cells to the site of injection, some of which then traffick the antigen to the draining lymph nodes to induce specific immune responses. However, the relationship between these recruited cells and induction of immune responses is not very clear. Depletion studies suggest that the role of recruited innate immune cells at the injection site is redundant in the generation of adaptive immune responses (McKee et al., 2009; Calabro et al., 2011). Interestingly, these studies were performed by depleting single cell populations. Identifying the role of a specific cell population in vivo is even more challenging due to complex environment at the injection site. Injection of adjuvants often leads to recruitment of a variety of cell populations and due to high redundancy in the immune system, other recruited cells may compensate for the depleted single cell population. In this regard, mice whose specific cell populations have been depleted were shown to produce cytokines and chemokines to recruit innate immune cells and activate $\mathrm{T}$ cells (Seubert et al., 2008; Calabro et al., 2011). Further studies are required to investigate the detailed relationship between recruited immune cells and adjuvant activity.

\section{ANTIGEN PRESENTATION}

Efficient antigen presentation by MHCs on APCs is important for the induction of adaptive immune response. It was thought that many adjuvants including alum, oil-based emulsions, and microparticles act by "targeting" antigens to APCs resulting in enhanced antigen presentation by MHC (Guéry et al., 1996; Schijns and Lavelle, 2011). Alum was shown to increase antigen uptake by DCs and alter the magnitude and duration of antigen presentation (Mannhalter et al., 1985; Morefield et al., 2005). Antigen adsorption on alum led to an increase in internalization of antigen (Morefield et al., 2005). Recent studies by Flach et al. (2011) have shown that alum does not enter DCs directly but rather delivers the antigen via abortive phagocytosis. In this regard, alum interacts with membrane lipids on DCs leading to lipid sorting, recruitment of ITAM containing molecules Syk and PI3 activation. These events eventually lead to uptake of antigen that is adsorbed on alum, DC activation, and MHC class II expression (Flach et al., 2011).

The role of adjuvant-induced increased antigen presentation in development of adaptive immunity has not been clearly evaluated. Hence, our knowledge is limited regarding the role of this adjuvant mechanism. Recently, Ghimire et al. (2012) investigated the impact of antigen presentation on alum adjuvanticity. In addition to confirming the ability of alum to increase the antigen internalization, the study also showed that alum plays an important role in reducing the rate of degradation of internalized antigen (Ghimire et al., 2012). Similarly, MF59 facilitated internalization of gD2 antigen from type 2 herpes simplex virus (HSV) by recruited APCs at the site of injection and increased phagocytosis in human PBMCs (Dupuis et al., 1999). Antigen size seems to play an important role in modulating the antigen presentation efficiency. Large lipid vesicles end up in early endosome/phagosomes and increases antigen presentation whereas smaller vesicles rapidly localize to late lysosomes leading to reduced antigen presentation (Brewer et al., 2004).

\section{ACTIVATION AND MATURATION OF DCs}

Activation of DCs is essential for induction of adaptive immune responses. Increased expression of MHC class II, activation marker CD86, and maturation marker CD83 leads to enhanced ability of APCs to induce T lymphocyte activation and differentiation (Coyle and Gutierrez-Ramos, 2001). Freund's complete adjuvant, lipopolysaccharide (LPS), liposomes, CpG-ODN, MF59, AS04, and $\alpha$-galactosylceramide ( $\alpha$-GAL) have all been shown to induce DC maturation to enhance adaptive immunity (De Smedt et al., 1996; De Becker et al., 2000; Copland et al., 2003; Fujii et al., 2003; Shah et al., 2003). Intra-peritoneal injection of OVA and alum led to uptake of antigen and maturation of DCs (Kool et al., 2008a). However, in vitro studies on human cells have shown that alum and MF59 failed to directly activate DCs but enhanced the surface expression of MHC class II and co-stimulatory molecules (CD83 and CD86) on monocytes, macrophages, and granulocytes that resulted in increased T cell proliferation (Sun et al., 2003; Seubert et al., 2008). Further, in vitro activation of DCs by alum has generated conflicting results. One study suggested that alum failed to induce maturation and antigen presentation (Sun et al., 2003) where as another study showed that the activation marker CD86 and antigen presentation was increased in DCs (Sokolovska et al., 2007). The source of alum may have been a contributing factor in the conflicting results.

AS04 has been shown to induce maturation of DCs (via TLR4), which then trafficks to the draining lymph nodes to activate antigen-specific T cells (Didierlaurent et al., 2009). Similarly, CpG induced up-regulation of CD40, CD54, CD80, CD86, and $\mathrm{MHC}$ class II molecules and antigen processing and presentation in plasmacytoid DCs (pDCs) (Krieg, 2002; Kerkmann et al., 2003). A novel class of TLR-independent adjuvants, mycobacterial cord factor trehalose-6-6-dimycolate (TDM) and TDB have 
been shown to directly activate DCs through the Fc $\gamma$ R-Syk-Card9Bcl10-Malt1 pathways, and up-regulates the expression of costimulatory molecules (Werninghaus et al., 2009). Microparticles such as Poly-lactic-co-glycolic acid (PLGA) did not induce costimulatory molecules expression on bone marrow derived DCs (BMDCs) but enhanced antigen presentation efficiency (Sun et al., 2003). DOTAP (1,2-dioleoyl-3-trimethylammonium-propane)based cationic liposomes have been shown to induce maturation of DCs through activation of MAPK (extracellular signal-regulated kinase and p38), leading to up-regulation of co-stimulatory molecules (Yan et al., 2007). Likewise, diC14-amidine (3tetradecylamino-tert-butyl-N-tetradecylpropion-amidine) based cationic liposomes up-regulates the expression of CD80 and CD86 on DCs through specific TLR4/MD2 ligation (Tanaka et al., 2008). Overall, adjuvants stimulate DC maturation and enhance the expression of MHC and co-stimulatory molecules, which is required for efficient $\mathrm{T}$ cell activation.

\section{ACTIVATION OF INFLAMMASOMES}

Innate immune cells express various pathogen-recognition receptors (PRRs) to recognize infectious agents. In recent years, various new families of PRRs have been identified including TLRs, C-type lectin-like receptors (CLRs), nucleotide oligomerization domain (NOD) like receptors (NLRs), and Retinoic acid-inducible gene1 (RIG-1) like receptors (RLRs). Many immunological adjuvants signal via PRRs or act as ligands for innate immune receptors (Table 2). In contrast to TLR agonists, particulate adjuvants are not recognized by specific PRRs but they still induce adaptive immune responses. The "danger" hypothesis was first advanced by Matzinger (1994), who proposed that apart from self/non-self discrimination against infection, danger signals from damaged cells can trigger activation of the immune system. Molecules associated with tissue damage such as uric acid, nucleotides, adenosine triphosphate (ATP), reactive oxygen intermediates, and cytokines are released at the injection site due to tissue damage (Shi et al., 2003). These non-infectious damage signals have now been named damage-associated molecular patterns (DAMPs) to distinguish them from pathogen-associated molecular patterns (PAMPs).

Particulate adjuvants cause local tissue damage and cell death at the injection site (Kool et al., 2008a). In addition, many adjuvants induce release of pro-inflammatory cytokines at the site of injection (Didierlaurent et al., 2009; Calabro et al., 2011; Awate et al., 2012). These damage signals trigger non-specific activation of the innate immune system, subsequently stimulating adaptive immunity. Recently inflammasomes have been one of the most widely investigated topics due to their potential role in adjuvant activity. The inflammasome belongs to the NLR family, which also includes various other receptors, such as the NODs (NOD15), NLRPs (NLRP1-14), NLRP1 (NAIP), NLRC4 (IPAF), and the major histocompatibility complex II transactivator (CIITA) (Martinon et al., 2009). Compared to others, NOD-like receptor family, pyrin-domain-containing 3 (NLRP3) is the most studied inflammasome receptor in regards to adjuvant mechanisms. NLRP3, also known as cryopyrin or NALP3 (NACHT, LRR, and PYD domainscontaining protein 3 ), is an intra-cytoplasmic multi-protein complex that consists of three components; a NLRP3 receptor, an apoptosis-associated speck-like protein containing a CARD (ASC) and a procaspase-1 (Schroder and Tschopp, 2010). Activation of NLRP3 inflammasome induces caspase- 1 activation, which in turn cleaves proforms of IL-1 $\beta$, IL-18, and IL-33 to their bioactive forms (Martinon et al., 2009). The NLRP3 inflammasome can be activated by various stimuli including DAMPs, environmental irritants such as asbestos and silica, metabolic stress, and UVB irradiation (Schroder and Tschopp, 2010). Apart from danger signals, inflammasomes can be activated by PAMPs such as bacterial flagellin through NLRC4 activation (Zhao et al., 2011).

Li et al. (2007) reported for the first time that alum-induced secretion of IL- $1 \beta$ and IL-18 was caspase- 1 dependent. Subsequent in vitro studies by various groups showed that activation of NLRP3 is required for alum-induced IL-1 $\beta$ and IL-18 secretion (Eisenbarth et al., 2008; Franchi and Nùñez, 2008; Hornung et al., 2008; Kool et al., 2008a). However, LPS priming to induce pro-IL-1 $\beta$ in APCs prior to alum stimulation was a pre-requisite for secretion of IL-1 $\beta$. Contrary to in vitro studies, the role of inflammasomes in the adjuvant activity of alum in vivo has yielded conflicting results. Using NLRP3, ASC and caspase-1 knockout mice, Eisenbarth et al. (2008) showed that the NLRP3 inflammasome is a crucial component in the adjuvant activity of alum. NLRP3, ASC, and caspase-1 knockout mice immunized with OVA adsorbed on alum, failed to induce antigen-specific antibody responses (Eisenbarth et al., 2008). Another study by Kool et al. (2008a) showed that alum-induced lower influx of inflammatory cells in the peritoneal cavity of NLRP3 deficient mice. They also showed that alum-mediated activation of adaptive immune responses was NLRP3-dependent (Kool et al., 2008a). Similar studies done by Li et al. (2008) showed that NLRP3 deficient mice injected with alum-adsorbed diphtheria toxoid or OVA vaccine elicited impaired levels of antigen-specific antibody responses. All these studies indicate that NLRP3 inflammasome is critical in the adjuvant activity of alum in vivo. In contrast, Franchi and Nùñez (2008) clearly showed that antigen-specific IgG production was not impaired in NLRP3 deficient mice following intra-peritoneal injection of human serum albumin (HSA) in the presence of alum. However, NLRP3 did affect alum-mediated cellular recruitment suggesting that inflammasomes might play an important role in activating innate immunity, but the contribution of inflammasomes in activation of adaptive immunity remains elusive. The conflicting results with regard to the role of inflammasomes in adjuvant activity of alum have been attributed to the differences in the nature of alum used in different studies, immunization protocols, and the mouse strains used (De Gregorio et al., 2008; Marrack et al., 2009).

To date, the ligand for NLRP3 has not been identified. Some theories proposed for alum-mediated activation of NLRP3 include phagosomal destabilization and release of cathepsin B, low intracellular potassium $(\mathrm{K}+)$ concentrations, and generation of reactive oxygen species (ROS) (Petrilli et al., 2007; Hornung et al., 2008; Kool et al., 2008a). It was proposed that a catabolic product of nucleotides, uric acid, and ATP released at the site of alum injection due to cell damage or necrosis act as danger signals for activation of NLRP3. Saturation of uric acid due to tissue damage forms monosodium ureate crystals (MSU). Phagocytosis of crystalline particles such as MSU or alum results in phagosomal destabilization and lysosomal rupture releasing the protease cathepsin B in the cytosol (Hornung et al., 2008). The released cathepsin B led to activation 
Table 2 | Innate immune receptors activated by vaccine adjuvants.

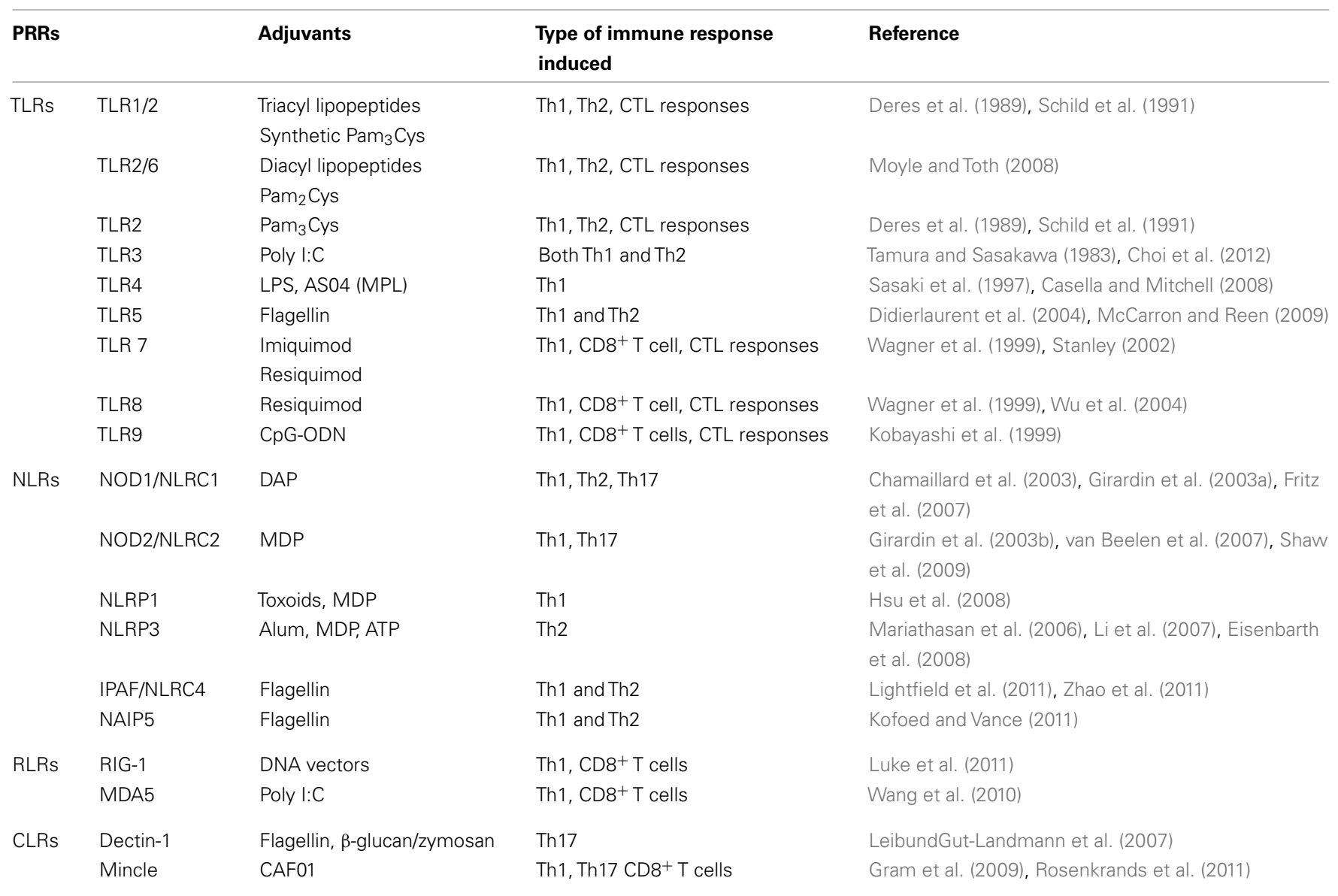

Pam3Cys, tri-palmitoyl-S-glyceryl cysteine; LPS, lipopolysaccharide; AS04, adjuvant system 04; MPL, monophosphoryl lipid A; CpG-ODN, cytidine-phosphate-guanosine oligodeoxynucleotides; Poly I:C, polyinosinic-polycytidylic acid; DAP, diaminopimelic acid; MDP, muramyl dipeptide; CAF01, cationic adjuvant formulation-01; TLR, tolllike receptor; NLR, NOD-like receptors; RLR, RIG-1 like receptors; $C L R, C$-type lectins; NOD, nucleotide-binding oligomerization domain; NLRP3, NOD-like receptor family, pyrin-domain-containing 3; IPAF, IL-13-converting enzyme protease-activating factor; NAIP, neuronal apoptosis inhibitory protein; RIG-1, retinoic acid-inducible gene-1; MDA5, melanoma differentiation associated gene 5 .

of NLRP3 and secretion of pro-inflammatory cytokines IL- $1 \beta$ and IL-18. Treatment of mice using uricase, a uric acid degrading enzyme, led to reduced cellular recruitment to draining lymph nodes in mice injected with alum (Kool et al., 2008a). Similarly, ATP released by the damaged cells at the injection site has been shown to indirectly activate NLRP3. Extracellular ATP triggered stimulation of purinergic $\mathrm{P}_{2} \mathrm{X}_{7}$ receptor, resulting in activation of cation channel for $\mathrm{K}+$ efflux and opening of pannexin-1 pore for entry of danger signals generated by alum, activate NLRP3 and subsequently caspase-1 (Solle et al., 2001; Petrilli et al., 2007). Further, blocking ROS using chemical scavengers abolished NLRP3 activation in response to MSU suggesting a link between NLRP3 activation and ROS generation (Dostert et al., 2008).

Recently, the role of the inflammasome in adjuvant activity of MF59 was evaluated (Ellebedy et al., 2011; Seubert et al., 2011). Two independent studies using NLRP3 deficient mice demonstrated that NLRP3 is not required for the adjuvant activity of MF59. However, an adaptor molecule required for the assembly of inflammasome, ASC was found to be crucial for MF59 adjuvant activity (Ellebedy et al., 2011). A recent study by Embry et al. (2011) showed that MPL failed to induce intra-cytoplasmic assembly of NLRP3 inflammasome leading to failure of caspase-1 activation and maturation of pro-inflammatory cytokines IL-1 $\beta$ and IL-18.

\section{CONCLUSION}

The ultimate goal of vaccination is to generate protection against disease causing pathogens. Protective immunity against different pathogens requires different immune responses that can be generated by using appropriate vaccine adjuvants. Therefore, a detailed knowledge of the mechanisms of action of adjuvants is very important in the rational design of vaccines. In recent years, considerable advances have been made in understanding the mechanisms of action of various adjuvants, particularly the activation of innate immunity via various mechanisms (Table 1 ). The future of vaccine adjuvant research is heading toward developing novel combination adjuvants that consist primarily of PRRs agonists and particulate adjuvants. While combining different adjuvants results in potent formulation that can enhance the quality and quantity of immune response against vaccine antigens, adjuvant combinations may also have more complex mechanisms of action. 
Safety is a major concern when it comes to adjuvant approval for human use. Detailed understanding of the mechanisms of action of adjuvants will provide some insight into their safety. In addition, since all of the adjuvants approved and currently in clinical trials are in vaccines administered by injection, there is a

\section{REFERENCES}

Awate, S., Wilson, H. L., Lai, K., Babiuk, L. A., and Mutwiri, G. (2012). Activation of adjuvant core response genes by the novel adjuvant PCEP. Mol. Immunol. 51, 292-303.

Brewer, J. M., Pollock, K. G. J., Tetley, L., and Russell, D. G. (2004). Vesicle size influences the trafficking, processing, and presentation of antigens in lipid vesicles. J. Immunol. 173, 6143-6150.

Bungener, L., Idema, J., ter Veer, W., Huckriede, A., Daemen, T., and Wilschut, J. (2002a). Virosomes in vaccine development: induction of cytotoxic $\mathrm{T}$ lymphocyte activity with virosome-encapsulated protein antigens. J. Liposome Res. 12, 155-163.

Bungener, L., Serre, K., Bijl, L., Leserman, L., Wilschut, J., Daemen, T., et al. (2002b). Virosome-mediated delivery of protein antigens to dendritic cells. Vaccine 20, 2287-2295.

Burrell, L. S., White, J. L., and Hem, S. L. (2000). Stability of aluminiumcontaining adjuvants during aging at room temperature. Vaccine 18 , 2188-2192.

Calabro, S., Tortoli, M., Baudner, B. C. Pacitto, A., Cortese, M., O’Hagan, D. T., et al. (2011). Vaccine adjuvants alum and MF59 induce rapid recruitment of neutrophils and monocytes that participate in antigen transport to draining lymph nodes. Vaccine 29, 1812-1823.

Casella, C., and Mitchell, T. (2008). Putting endotoxin to work for us: monophosphoryl lipid A as a safe and effective vaccine adjuvant. Cell. Mol. Life Sci. 65, 3231-3240.

Chamaillard, M., Hashimoto, M., Horie, Y., Masumoto, J., Qiu, S., Saab, L., et al. (2003). An essential role for NOD1 in host recognition of bacterial peptidoglycan containing diaminopimelic acid. Nat. Immunol. 4, 702-707.

Choi, J. P., Kim, Y. S., Kim, O. Y., Kim, Y. M., Jeon, S. G., Roh, T. Y., et al. (2012). TNF-alpha is a key mediator in the development of Th2 cell response to inhaled allergens induced by a viral PAMP double-stranded RNA. Allergy 67, 1138-1148.

Copland, M. J., Baird, M. A., Rades, T., McKenzie, J. L., Becker, B., Reck, F., et al. (2003). Liposomal delivery of antigen to human dendritic cells. Vaccine 21, 883-890.

Cox, J. C., and Coulter, A. R. (1997). Adjuvants-a classification and review of their modes of action. Vaccine 15, 248-256.

Coyle, A. J., and Gutierrez-Ramos, J.-C. (2001). The expanding B7 superfamily: increasing complexity in costimulatory signals regulating T cell function. Nat. Immunol. 2, 203-209.

De Becker, G. V., Moulin, V. R., Pajak, B., Bruck, C., Francotte, M., Thiriart, C., et al. (2000). The adjuvant monophosphoryl lipid A increases the function of antigenpresenting cells. Int. Immunol. 12, 807-815.

De Gregorio, E., Tritto, E., and Rappuoli, R. (2008). Alum adjuvanticity: unraveling a century old mystery. Eur. J. Immunol. 38, 2068-2071.

De Smedt, T., Pajak, B., Muraille, E., Lespagnard, L., Heinen, E., De Baetselier, P., et al. (1996). Regulation of dendritic cell numbers and maturation by lipopolysaccharide in vivo. J. Exp. Med. 184, 1413-1424.

Deres, K., Schild, H., Wiesmuller, K.H., Jung, G., and Rammensee, H.-G. (1989). In vivo priming of virusspecific cytotoxic $\mathrm{T}$ lymphocytes with synthetic lipopeptide vaccine. Nature 342, 561-564.

Didierlaurent, A., Ferrero, I., Otten, L. A., Dubois, B., Reinhardt, M., Carlsen, H., et al. (2004). Flagellin promotes myeloid differentiation factor 88-dependent development of Th2-type response. J. Immunol. 172, 6922-6930.

Didierlaurent, A. M., Morel, S., Lockman, L., Giannini, S. L., Bisteau, M., Carlsen, H., et al. (2009). AS04, an aluminum salt- and TLR4 agonistbased adjuvant system, induces a transient localized innate immune response leading to enhanced adaptive immunity. J. Immunol. 183, 6186-6197.

Dostert, C., Pétrilli, V., Van Bruggen, R., Steele, C., Mossman, B. T., and Tschopp, J. (2008). Innate immune activation through Nalp3 inflammasome sensing of asbestos and silica. Science 320, 674-677.

Dupuis, M., Denis-Mize, K., LaBarbara, A., Peters, W., Charo, I. F., McDonald, D. M., et al. (2001). Immunization with the adjuvant

need to identify and develop good mucosal adjuvants. In the coming years, we hope to learn more details of the various mechanisms of action of adjuvants, which will be valuable in rational vaccine design and hopefully lead to approval of new adjuvants for use in vaccines for humans.

MF59 induces macrophage trafficking and apoptosis. Eur. J. Immunol. 31, 2910-2918.

Dupuis, M., McDonald, D. M., and Ott, G. (1999). Distribution of adjuvant MF59 and antigen gD2 after intramuscular injection in mice. Vaccine 18, 434-439.

Eisenbarth, S. C., Colegio, O. R. O'Connor, W., Sutterwala, F. S., and Flavell, R. A. (2008). Crucial role for the Nalp3 inflammasome in the immunostimulatory properties of aluminium adjuvants. Nature 453, 1122-1126.

Ellebedy, A. H., Lupfer, C., Ghoneim, H. E., DeBeauchamp, J., Kanneganti, T.-D., and Webby, R. J. (2011). Inflammasome-independent role of the apoptosis-associated speck-like protein containing CARD (ASC) in the adjuvant effect of MF59. Proc. Natl. Acad. Sci. U.S.A. 108 2927-2932.

Embry, C. A., Franchi, L., Nunez, G., and Mitchell, T. C. (2011). Mechanism of impaired NLRP3 inflammasome priming by monophosphoryl lipid A. Sci. Signal. 4, ra28.

Flach, T. L., Ng, G., Hari, A., Desrosiers, M. D., Zhang, P., Ward, S. M., et al. (2011). Alum interaction with dendritic cell membrane lipids is essential for its adjuvanticity. Nat. Med. 17, 479-487.

Franchi, L., and Nùñez, G. (2008). The Nlrp3 inflammasome is critical for aluminium hydroxide-mediated ILlbeta secretion but dispensable for adjuvant activity. Eur. J. Immunol. 38, 2085-2089.

Fraser, C. K., Diener, K. R., Brown, M. P., and Hayball, J. D. (2007). Improving vaccines by incorporating immunological coadjuvants. Expert Rev. Vaccines 6, 559-578.

Fritz, J. H., Le Bourhis, L., Sellge, G. Magalhaes, J. G., Fsihi, H., Kufer, T. A., et al. (2007). Nod1-mediated innate immune recognition of peptidoglycan contributes to the onset of adaptive immunity. Immunity 26 , 445-459.

Fujii, S.-I., Shimizu, K., Smith, C., Bonifaz, L., and Steinman, R. M. (2003). Activation of natural killer $\mathrm{T}$ cells by $\alpha$-Galactosylceramide rapidly induces the full maturation of dendritic cells in vivo and thereby acts as an adjuvant for combined CD4 and CD8 T cell immunity to a coadministered protein. J. Exp. Med. 198, 267-279.

Gavin, A. L., Hoebe, K., Duong, B., Ota, T., Martin, C., Beutler, B., et al. (2006). Adjuvant-enhanced antibody responses in the absence of tolllike receptor signaling. Science 314, 1936-1938.

Ghimire, T. R., Benson, R. A., Garside, P., and Brewer, J. M. (2012). Alum increases antigen uptake, reduces antigen degradation and sustains antigen presentation by DCs in vitro. Immunol. Lett. 147, 55-62.

Girardin, S. E., Boneca, I. G., Carneiro, L. A. M., Antignac, A., Jéhanno M., Viala, J., et al. (2003a). Nod1 detects a unique muropeptide from gram-negative bacterial peptidoglycan. Science 300, 1584-1587.

Girardin, S. E., Boneca, I. G., Viala, J. R. M., Chamaillard, M., Labigne, A. S., Thomas, G., et al. (2003b). Nod2 is a general sensor of peptidoglycan through muramyl dipeptide (MDP) detection. J. Biol. Chem. 278, 8869-8872.

Glenny, A. T., Pope, C. G., Waddington, H., and Wallace, V. (1926). The antigenic value of toxoid precipitated by potassium-alum. J. Pathol. Bacteriol. 29, 38-45.

Glück, R., Mischler, R., Brantschen, S., Just, M., Althaus, B., and Cryz, S. J. (1992). Immunopotentiating reconstituted influenza virus virosome vaccine delivery system for immunization against hepatitis A. J. Clin. Invest. 90, 2491-2495.

Goto, N., and Akama, K. (1982). Histopathological studies of reactions in mice injected with aluminum-adsorbed tetanus toxoid. Microbiol. Immunol. 26, 1121-1132.

Goto, N., Kato, H., Maeyama, J.-I., Shibano, M., Saito, T., Yamaguchi, J., et al. (1997). Local tissue irritating effects and adjuvant activities of calcium phosphate and aluminium hydroxide with different physical properties. Vaccine 15, 1364-1371.

Gram, G. J., Karlsson, I., Agger, E. M., Andersen, P., and Fomsgaard A. (2009). A novel liposome-based adjuvant CAF01 for induction of CD8(+) cytotoxic T-lymphocytes (CTL) to HIV-1 minimal CTL peptides in HLA-A $* 0201$ transgenic mice. PLoS ONE 4:e6950. doi:10.1371/journal.pone.0006950 
Grun, J. L., and Maurer, P. H. (1989). Different $\mathrm{T}$ helper cell subsets elicited in mice utilizing two different adjuvant vehicles: the role of endogenous interleukin 1 in proliferative responses. Cell. Immunol. 121, 134-145.

Guéry, J. C., Ria, F., and Adorini, L. (1996). Dendritic cells but not B cells present antigenic complexes to class II-restricted T cells after administration of protein in adjuvant. J. Exp. Med. 183, 751-757.

Guy, B. (2007). The perfect mix: recent progress in adjuvant research. Nat. Rev. Microbiol. 5, 505-517.

Henriksen-Lacey, M., Bramwell, V. W., Christensen, D., Agger, E.-M., Andersen, P., and Perrie, Y. (2010). Liposomes based on dimethyldioctadecylammonium promote a depot effect and enhance immunogenicity of soluble antigen. J. Control Release. $142,180-186$.

Herbert, W. J. (1968). The mode of action of mineral-oil emulsion adjuvants on antibody production in mice. Immunology 14, 301-318.

Hoebe, K., Janssen, E., and Beutler, B. (2004). The interface between innate and adaptive immunity. Nat. Immunol. 5, 971-974.

Hornung, V., Bauernfeind, F., Halle, A., Samstad, E. O., Kono, H., Rock, K. L., et al. (2008). Silica crystals and aluminum salts activate the NALP3 inflammasome through phagosomal destabilization. Nat. Immunol. 9, 847-856.

Hsu, L.-C., Ali, S. R., McGillivray, S., Tseng, P.-H., Mariathasan, S., Humke, E. W., et al. (2008). A NOD2-NALP1 complex mediates caspase-1-dependent IL-1 $\beta$ secretion in response to Bacillus anthracis infection and muramyl dipeptide. Proc. Natl. Acad. Sci. U.S.A. 105, 7803-7808.

Hutchison, S., Benson, R. A., Gibson, V. B., Pollock, A. H., Garside, P., and Brewer, J. M. (2012). Antigen depot is not required for alum adjuvanticity. FASEB J. 26, 1272-1279.

Iyer, S., HogenEsch, H., and Hem, S. L. (2003). Relationship between the degree of antigen adsorption to aluminum hydroxide adjuvant in interstitial fluid and antibody production. Vaccine 21, 1219-1223.

Janeway, C. A. (1989). Approaching the asymptote? Evolution and revolution in immunology. Cold Spring Harb. Symp. Quant. Biol. 54, 1-13.

Jordan, M. B., Mills, D. M., Kappler, J., Marrack, P., and Cambier, J. C. (2004). Promotion of $B$ cell immune responses via an alum-induced myeloid cell population. Science 304, 1808-1810.

Kerkmann, M., Rothenfusser, S., Hornung, V., Towarowski, A., Wagner, M., Sarris, A., et al. (2003). Activation with $\mathrm{CpG}-\mathrm{A}$ and $\mathrm{CpG}-$ $\mathrm{B}$ oligonucleotides reveals two distinct regulatory pathways of type I IFN synthesis in human plasmacytoid dendritic cells. J. Immunol. 170, 4465-4474.

Khoshnejad, M., Young, P. R., Toth, I., and Minchin, R. F. (2007). Modified influenza virosomes: recent advances and potential in gene delivery. Curr. Med. Chem. 14, 3152-3156.

Klaschik, S., Tross, D., and Klinman, D. M. (2009). Inductive and suppressive networks regulate TLR9dependent gene expression in vivo. J. Leukoc. Biol. 85, 788-795.

Klaschik, S., Tross, D., Shirota, H., and Klinman, D. M. (2010). Shortand long-term changes in gene expression mediated by the activation of TLR9. Mol. Immunol. 47, 1317-1324.

Klinman, D. M., Yi, A. K., Beaucage, S. L., Conover, J., and Krieg, A. M. (1996). CpG motifs present in bacteria DNA rapidly induce lymphocytes to secrete interleukin 6 , interleukin 12, and interferon gamma. Proc. Natl. Acad. Sci. U.S.A. 93, 2879-2883.

Kobayashi, H., Horner, A. A., Takabayashi, K., Nguyen, M.-D., Huang, E., Cinman, N., et al. (1999). Immunostimulatory DNA prepriming: a novel approach for prolonged Th1-biased immunity. Cell. Immunol. 198, 69-75.

Kofoed, E. M., and Vance, R. E. (2011). Innate immune recognition of bacterial ligands by NAIPs determines inflammasome specificity. Nature 477, 592-595.

Kool, M., Pétrilli, V., De Smedt, T., Rolaz, A., Hammad, H., van Nimwegen, M., et al. (2008a). Cutting edge: alum adjuvant stimulates inflammatory dendritic cells through activation of the NALP3 inflammasome. J. Immunol. 181, 3755-3759.

Kool, M., Soullié, T., van Nimwegen, M., Willart, M. A. M., Muskens, F., Jung, S., et al. (2008b). Alum adjuvant boosts adaptive immunity by inducing uric acid and activating inflammatory dendritic cells. J. Exp. Med. 205, 869-882.

Korsholm, K. S., Petersen, R. V., Agger, E. M., and Andersen, P. (2010). Thelper 1 and T-helper 2 adjuvants induce distinct differences in the magnitude, quality and kinetics of the early inflammatory response at the site of injection. Immunology 129, 75-86.

Kreuter, J. (1988). Possibilities of using nanoparticles as carriers for drugs and vaccines. J. Microencapsul. 5 , 115-127.

Krieg, A. M. (2002). CpG motifs in bacterial DNA and their immune effects. Annu. Rev. Immunol. 20, 709-760.

LeibundGut-Landmann, S., Grosz, O. Robinson, M. J., Osorio, F., Slack, E. C., Tsoni, S. V., et al. (2007). Syk- and CARD9-dependent coupling of innate immunity to the induction of $\mathrm{T}$ helper cells that produce interleukin 17. Nat. Immunol. 8, 630-638.

Li, H., Nookala, S., and Re, F. (2007). Aluminum hydroxide adjuvants activate caspase- 1 and induce IL-1 $\beta$ and IL-18 release. J. Immunol. 178, 5271-5276.

Li, H., Willingham, S. B., Ting, J. P.Y., and Re, F. (2008). Cutting edge: inflammasome activation by alum and alum's adjuvant effect are mediated by NLRP3. J. Immunol. 181, 17-21.

Lightfield, K. L., Persson, J., Trinidad, N. J., Brubaker, S. W., Kofoed, E. M. Sauer, J.-D., et al. (2011). Differential requirements for NAIP5 in activation of the NLRC4 inflammasome. Infect. Immun. 79, 1606-1614.

Luke, J. M., Simon, G. G., Soderholm, J., Errett, J. S., August, J. T., Gale, M., et al. (2011). Coexpressed RIG-I agonist enhances humoral immune response to influenza virus DNA vaccine. J. Virol. 85, 1370-1383.

Mannhalter, J. W., Neychev, H. O. Zlabinger, G. J., Ahmad, R., and Eibl, M. M. (1985). Modulation of the human immune response by the non-toxic and non-pyrogenic adjuvant aluminium hydroxide: effect on antigen uptake and antigen presentation. Clin. Exp. Immunol. 61, 143-151.

Mariathasan, S., Weiss, D. S., Newton, K., McBride, J., O’Rourke, K., RooseGirma, M., et al. (2006). Cryopyrin activates the inflammasome in response to toxins and ATP. Nature 440, 228-232.

Marrack, P., McKee, A. S., and Munks, M. W. (2009). Towards an understanding of the adjuvant action of aluminium. Nat. Rev. Immunol. 9 , 287-293.

Martinon, F., Mayor, A., and Tschopp, J. (2009). The inflammasomes: guardians of the body. Annu. Rev. Immunol. 27, 229-265.

Matzinger, P. (1994). Tolerance, danger, and the extended family. Annu. Rev. Immunol. 12, 991-1045.
McCarron, M., and Reen, D. J. (2009). Activated human neonatal CD8+ $\mathrm{T}$ cells are subject to immunomodulation by direct TLR2 or TLR5 stimulation. J. Immunol. 182, 55-62.

McKee, A. S., Munks, M. W., MacLeod, M. K. L., Fleenor, C. J., Van Rooijen, N., Kappler, J. W., et al. (2009). Alum induces innate immune responses through macrophage and mast cell sensors, but these sensors are not required for alum to act as an adjuvant for specific immunity. $J$. Immunol. 183, 4403-4414.

Morefield, G. L., Sokolovska, A., Jiang, D., HogenEsch, H., Robinson, J. P., and Hem, S. L. (2005). Role of aluminum-containing adjuvants in antigen internalization by dendritic cells in vitro. Vaccine 23, 1588-1595.

Morein, B., and Bengtsson, K. L. V. (1999). Immunomodulation by iscoms, immune stimulating complexes. Methods 19, 94-102.

Morel, S., Didierlaurent, A., Bourguignon, P., Delhaye, S., Baras, B., Jacob, V., et al. (2011). Adjuvant system AS03 containing $\alpha$-tocopherol modulates innate immune response and leads to improved adaptive immunity. Vaccine 29, 2461-2473.

Mosca, F., Tritto, E., Muzzi, A., Monaci, E., Bagnoli, F., Iavarone, C., et al. (2008). Molecular and cellular signatures of human vaccine adjuvants. Proc. Natl. Acad. Sci. U.S.A. 105, 10501-10506.

Moyle, P. M., and Toth, I. (2008). Selfadjuvanting lipopeptide vaccines. Curr. Med. Chem. 15, 506-516.

Osebold, J. W. (1982). Mechanisms of action by immunologic adjuvants. $J$. Am. Vet. Med. Assoc. 181, 983-987.

Ott, G., Barchfeld, G. L., Chernoff, D. Radhakrishnan, R., van Hoogevest, P., and Van Nest, G. (1995). MF59. Design and evaluation of a safe and potent adjuvant for human vaccines. Pharm. Biotechnol. 6, 277-296.

Pashine, A., Valiante, N. M., and Ulmer, J. B. (2005). Targeting the innate immune response with improved vaccine adjuvants. Nat. Med. 11, 63-68.

Petrilli, V., Papin, S., Dostert, C., Mayor, A., Martinon, F., and Tschopp, J. (2007). Activation of the NALP3 inflammasome is triggered by low intracellular potassium concentration. Cell Death Differ. 14, 1583-1589.

Ramanathan, V. D., Badenoch-Jones, P., and Turk, J. L. (1979). Complement activation by aluminium and zirconium compounds. Immunology 37, 881-888. 
Ramon, G. (1924). Sur la toxine et sur I'anatoxine diphtheriques. Ann. Inst. Pasteur 38, 1-10.

Rimaniol, A.-C. C., Gras, G., Verdier, F. O., Capel, F., Grigoriev, V. B., Porcheray, F., et al. (2004). Aluminum hydroxide adjuvant induces macrophage differentiation towards a specialized antigenpresenting cell type. Vaccine 22, 3127-3135.

Rosenkrands, I., Vingsbo-Lundberg, C., Bundgaard, T. J., Lindenström, T., Enouf, V., van der Werf, S., et al. (2011). Enhanced humoral and cell-mediated immune responses after immunization with trivalent influenza vaccine adjuvanted with cationic liposomes. Vaccine 29, 6283-6291.

Sasaki, S., Tsuji, T., Hamajima, K., Fukushima, J., Ishii, N., Kaneko, T., et al. (1997). Monophosphoryl lipid A enhances both humoral and cell-mediated immune responses to DNA vaccination against human immunodeficiency virus type 1 . Infect. Immun. 65, 3520-3528.

Schijns, V. E. J. C. (2000). Immunological concepts of vaccine adjuvant activity: commentary. Curr. Opin. Immunol. 12, 456-463.

Schijns, V. E. J. C., and Lavelle, E. C. (2011). Trends in vaccine adjuvants. Expert Rev. Vaccines 10, 539-550.

Schild, H., Deres, K., Wiesmüller, K.H., Jung, G., and Rammensee, H.-G. (1991). Efficiency of peptides and lipopeptides for in vivo priming of virus-specific cytotoxic T cells. Eur. J. Immunol. 21, 2649-2654.

Schroder, K., and Tschopp, J. (2010). The inflammasomes. Cell 140, 821-832.

Serre, K., Mohr, E., Toellner, K. M., Cunningham, A. F., Granjeaud, S., Bird, R., et al. (2008). Molecular differences between the divergent responses of ovalbumin-specific CD4 $\mathrm{T}$ cells to alum-precipitated ovalbumin compared to ovalbumin expressed by Salmonella. Mol. Immunol. 45, 3558-3566.
Seubert, A., Calabro, S., Santini, L., Galli, B., Genovese, A., Valentini, S., et al. (2011). Adjuvanticity of the oil-inwater emulsion MF59 is independent of Nlrp3 inflammasome but requires the adaptor protein MyD88. Proc. Natl. Acad. Sci. U.S.A. 108, 11169-11174.

Seubert, A., Monaci, E., Pizza, M., O'Hagan, D. T., and Wack, A. (2008). The adjuvants aluminum hydroxide and MF59 induce monocyte and granulocyte chemoattractants and enhance monocyte differentiation toward dendritic cells. J. Immunol. 180, 5402-5412.

Shah, J. A., Darrah, P. A., Ambrozak, D. R., Turon, T. N., Mendez, S., Kirman, J., et al. (2003). Dendritic cells are responsible for the capacity of CpG oligodeoxynucleotides to act as an adjuvant for protective vaccine immunity against Leishmania major in mice. J. Exp. Med. 198, 281-291.

Shaw, M. H., Reimer, T., SanchezValdepenas, C., Warner, N., Kim, Y.-G., Fresno, M., et al. (2009). T cell-intrinsic role of Nod2 in promoting type 1 immunity to Toxoplasma gondii. Nat. Immunol. 10, 1267-1274.

Shi, Y., Evans, J. E., and Rock, K. L. (2003). Molecular identification of a danger signal that alerts the immune system to dying cells. Nature 425, 516-521.

Singh, M., and O'Hagan, D. T. (2003). Recent advances in veterinary vaccine adjuvants. Int. J. Parasitol. 33, 469-478.

Siskind, G. W., and Benacerraf, B. (1969). Cell selection by antigen in the immune response. $A d v$. Immunol. 10, 1-50.

Sokolovska, A., Hem, S. L., and HogenEsch, H. (2007). Activation of dendritic cells and induction of $\mathrm{CD} 4+\mathrm{T}$ cell differentiation by aluminum-containing adjuvants. Vaccine 25, 4575-4585.

Solle, M., Labasi, J., Perregaux, D. G., Stam, E., Petrushova, N., Koller, B. H., et al. (2001). Altered cytokine production in mice lacking P2 $\times 7$ receptors. J. Biol. Chem. 276 , 125-132.

Stanley, M. A. (2002). Imiquimod and the imidazoquinolines: mechanism of action and therapeutic potential. Clin. Exp. Dermatol. 27, 571-577.

Sun, H., Pollock, K. G. J., and Brewer, J. M. (2003). Analysis of the role of vaccine adjuvants in modulating dendritic cell activation and antigen presentation in vitro. Vaccine 21 849-855.

Tamura, M., and Sasakawa, S. (1983). Induction of human leukocyte interferon by heat-treated poly I: poly C. Biochem. Biophys. Res. Commun. 110, 851-858.

Tanaka, T., Legat, A., Adam, E., Steuve, J. Gatot, J.-S., Vandenbranden, M., et al. (2008). DiC14-amidine cationic liposomes stimulate myeloid dendritic cells through Toll-like receptor 4. Eur. J. Immunol. 38, 1351-1357.

van Beelen, A. J., Zelinkova, Z., Taanman-Kueter, E. W., Muller, F. J., Hommes, D. W., Zaat, S. A. J., et al. (2007). Stimulation of the intracellular bacterial sensor NOD2 programs dendritic cells to promote interleukin-17 production in human memory $\mathrm{T}$ cells. Immunity 27, 660-669.

Wagner, T. L., Ahonen, C. L., Couture, A M., Gibson, S. J., Miller, R. L., Smith, R. M., et al. (1999). Modulation of TH1 and TH2 cytokine production with the immune response modifiers, R-848 and imiquimod. Cell. Immunol. 191, 10-19.

Wang, H.-B., and Weller, P. F. (2008). Pivotal advance: eosinophils mediate early alum adjuvant-elicited B cell priming and IgM production. J. Leukoc. Biol. 83, 817-821.

Wang, Y., Cella, M., Gilfillan, S., and Colonna, M. (2010). Cutting edge: polyinosinic:polycytidylic acid boosts the generation of memory CD8 $\mathrm{T}$ cells through melanoma differentiation-associated protein 5 expressed in stromal cells. J. Immunol. 184, 2751-2755.
Werninghaus, K., Babiak, A., Groß, O., Hölscher, C., Dietrich, H., Agger, E. M., et al. (2009). Adjuvanticity of a synthetic cord factor analogue for subunit Mycobacterium tuberculosis vaccination requires FcR $\gamma$-Syk-Card9-dependent innate immune activation. J. Exp. Med. 206, 89-97.

Wu, J. J., Huang, D. B., and Tyring, S. K. (2004). Resiquimod: a new immune response modifier with potential as a vaccine adjuvant for Th1 immune responses. Antiviral Res. 64, 79-83.

Yan, W., Chen, W., and Huang, L. (2007). Mechanism of adjuvant activity of cationic liposome: phosphorylation of a MAP kinase, ERK and induction of chemokines. Mol. Immunol. 44, 3672-3681.

Zhao, Y., Yang, J., Shi, J., Gong, Y.-N., Lu, Q., Xu, H., et al. (2011). The NLRC4 inflammasome receptors for bacterial flagellin and type III secretion apparatus. Nature 477, 596-600.

Conflict of Interest Statement: The authors declare that the research was conducted in the absence of any commercial or financial relationships that could be construed as a potential conflict of interest.

Received: 20 March 2013; paper pending published: 14 April 2013; accepted: 29 April 2013; published online: 16 May 2013.

Citation: Awate S, Babiuk LA and Mutwiri G (2013) Mechanisms of action of adjuvants. Front. Immunol. 4:114. doi: 10.3389/fimmu.2013.00114

This article was submitted to Frontiers in Immunotherapies and Vaccines, a specialty of Frontiers in Immunology. Copyright (c) 2013 Awate, Babiuk and Mutwiri. This is an open-access article distributed under the terms of the Creative Commons Attribution License, which permits use, distribution and reproduction in other forums, provided the original authors and source are credited and subject to any copyright notices concerning any third-party graphics etc. 